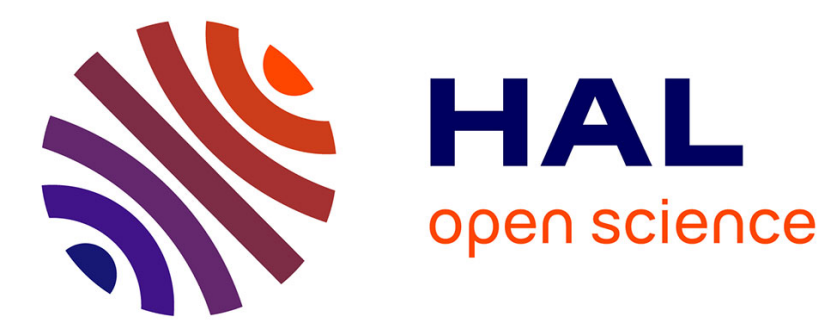

\title{
Développement d'une nouvelle méthode de suivi de la corrosion par des capteurs sans fil basés sur la technologie RFID
}

\author{
Rania Khalifeh, Stéphane Rioual, Benoit Lescop
}

\section{- To cite this version:}

Rania Khalifeh, Stéphane Rioual, Benoit Lescop. Développement d'une nouvelle méthode de suivi de la corrosion par des capteurs sans fil basés sur la technologie RFID. 7ème Colloque Interdisciplinaire en Instrumentation, Jan 2016, Saint-Nazaire, France. hal-01280227

\section{HAL Id: hal-01280227 \\ https://hal.science/hal-01280227}

Submitted on 29 Feb 2016

HAL is a multi-disciplinary open access archive for the deposit and dissemination of scientific research documents, whether they are published or not. The documents may come from teaching and research institutions in France or abroad, or from public or private research centers.
L'archive ouverte pluridisciplinaire HAL, est destinée au dépôt et à la diffusion de documents scientifiques de niveau recherche, publiés ou non, émanant des établissements d'enseignement et de recherche français ou étrangers, des laboratoires publics ou privés. 


\title{
Développement d'une nouvelle méthode de suivi de la corrosion par des capteurs sans fil basés sur la technologie RFID
}

\author{
R. Khalifeh¹,2, B. Lescop¹, F. Gallée², G. Le Roux¹, et S. Rioual ${ }^{1}$ \\ ${ }^{1}$ Laboratoire de Magnétisme de Bretagne, Université de Bretagne Occidentale, 6 av. Le \\ Gorgeu; 29285 Brest Cedex, France \\ 2 Lab-STICC/MOM, Telecom Bretagne, Technopôle Brest-Iroise, CS 83818, 29238 Brest
}

Cedex, France

\begin{abstract}
Wireless and fully energetically passive corrosion sensors inspired from the chipless Radiofrequency Identification (RFID) technology are presented for the monitoring of degradation of materials. The device consists of a reader and a remote passive sensor. The study presents two radiofrequency corrosion sensitive resonators which can be integrated in such device. The first is sensitive to corrosion potential $E_{\text {corr }}$ with respect to a reference electrode. The radiofrequency characterization of this resonator demonstrates unambiguously the sensitivity of the method to the passivation of steel in sea water and to the degradation of organic coatings. In a second part, the sensing element is modified to provide information on electrical potential and current.
\end{abstract}

\section{Introduction}

La corrosion est un processus électrochimique qui dégrade la plupart des matériaux, et qui de ce fait, présente un fort impact financier au niveau des opérations de maintenance des infrastructures dans de nombreux domaines d'activités : transport, énergie, construction, ... II est donc crucial de détecter et quantifier avec précision l'évolution de la corrosion par l'utilisation de capteurs ou de réseaux de capteurs. Ceci est notamment vrai dans le cadre des activités marines et portuaires, pour lesquelles les conditions environnementales peuvent être considérées comme sévères.

Les méthodes électrochimiques sont fortement utilisées pour contrôler l'évolution de la dégradation des revêtements organiques protégeant les structures métalliques. La spectroscopie d'impédance (Electrochemical Impedance Spectroscopy (EIS)) permet, en particulier, l'évaluation de la performance des revêtements organiques, la présence de défauts au sein de ces revêtements ou encore la diffusion de l'eau. Cette technique donne également accès au contrôle de la formation de biofilms sur de l'acier immergé en eau de mer. Malgré le succès de cette méthode, elle nécessite l'utilisation d'instruments de mesure lourds et volumineux tels que les potentiostats, rendant ainsi difficile les mesures in-situ. Récemment, des mini-potentiostats de faibles tailles et fonctionnant "sans fil » ont été proposés comme solution au problème [1,2]. Dans ce cas, le potentiostat fonctionne grâce à 
l'utilisation d'une batterie interne. Son intégration aux structures mécaniques n'est alors faisable que sous réserve de pouvoir régulièrement changer la batterie. D'autres méthodes de contrôle de la corrosion existent. Récemment, Wilson et al. [3] ont proposé d'insérer entre un substrat métallique et un revêtement, des microfils conducteurs. Lors de la dégradation du revêtement, la résistance électrique des microfils est modifiée rendant ainsi possible le contrôle de la dégradation du polymère. Cette méthode est proche des méthodes résistives utilisées en corrosion atmosphérique qui permettent de déterminer la vitesse de corrosion des métaux dans le cas de corrosion uniforme [4]. De la même façon que précédemment, il est possible de déporter le capteur via l'utilisation de batteries et du dispositif électronique associé (générateur de courant, transmission sans fil..).

Dans ce contexte, il existe un réel intérêt à proposer des capteurs sans fil totalement passifs énergétiquement (sans batterie), de faibles tailles et pouvant être intégrés à des structures mécaniques. L'avantage de ce type de capteur est alors de pouvoir être placé dans une région inaccessible, où le remplacement de la batterie est impossible ou délicat (intégration dans du béton, sous revêtement,...). L'ensemble de ces critères répond à la technologie RFID (Radio-Frequency Identification) fortement utilisée de nos jours pour réaliser des tâches d'identification dans de nombreux domaines. Dans ce cas, un lecteur envoie une onde électromagnétique via des antennes vers un microcontrôleur. Celui-ci la reçoit, ce qui lui permet d'acquérir de l'énergie et de renvoyer son identifiant au lecteur. L'objectif de cette étude concerne le développement de capteurs de corrosion innovants inspirés de cette technologie. Récemment, deux solutions basées sur ce principe ont été proposées $[5,6]$. Elles reposent sur l'évolution de l'amplitude du signal entre le lecteur et le microcontrôleur. Pour cela un élément sensible à la corrosion est intégré au niveau des antennes. Cependant, malgré la simplicité de la méthode, elle est fortement dépendante du fonctionnement du microcontrôleur et il n'est donc pas aisé de remonter à une information fiable sur le niveau de corrosion du métal composant l'élément sensible. Afin de lever ce verrou technologique, nous avons proposé récemment de considérer une technologie RFID sans microcontrôleur. Pour cela, nous nous basons sur l'architecture proposée par Preradovic et Karmakar [7] présentée sur la figure 1.

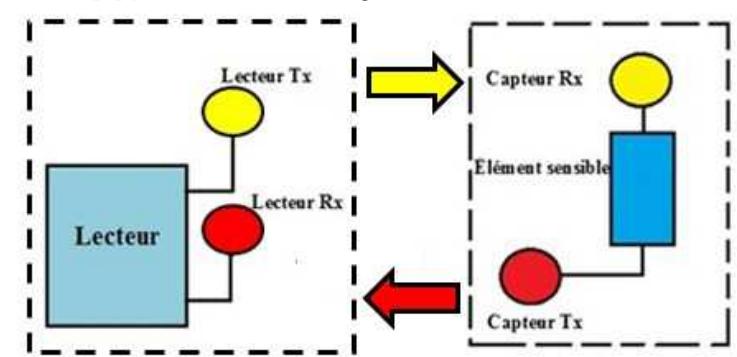

Figure 1: Schéma du capteur de corrosion passif associé au lecteur

Comme dans la technologie RFID avec microcontrôleur, le dispositif est constitué d'un lecteur et d'un capteur. Le capteur présente une partie sensible à la corrosion reliée à deux antennes $(\mathrm{Rx}$ et $\mathrm{Tx})$ permettant de recevoir et de réémettre le signal. Des antennes à polarisation croisées sont choisies afin d'isoler les signaux $\mathrm{Rx}$ et $\mathrm{Tx}$ du lecteur. La technologie micro-onde retenue pour ce type de capteur est une technologie micro-ruban, ce qui permet une connexion simple de la partie sensible aux antennes. Dans le passé, nous 
avons proposé différents types de capteurs basés sur cette technologie. En particulier, nous avons développé une partie sensible permettant de contrôler et de mesurer la perte de masse d'un élément métallique via l'utilisation d'une ligne micro-ruban [8] ou d'un résonateur hyperfréquence $[9,10]$. Dans ces deux cas, les résultats donnent accès, comme pour les capteurs résistifs de corrosion, à la vitesse de corrosion en cas de corrosion uniforme. Ils permettent également de différencier les mécanismes de corrosion : uniforme ou localisée. Cependant, la détermination de la perte de masse n'est pas le seul paramètre pertinent pour le contrôle de la dégradation des matériaux. En particulier, lorsqu'un métal est immergé dans un électrolyte, il présente un potentiel spécifique par rapport à une électrode de référence, le potentiel de corrosion $E_{\text {corr. }}$. Ce paramètre peut être utilisé comme premier indicateur pour suivre l'évolution du métal au cours du temps, notamment dans le cas du développement de biofilms sur de l'acier en eau de mer [11]. Dans ce cadre, nous présentons dans cette étude le développement d'une fonction hyperfréquence sensible à $E_{\text {corr }}$ et intégrable dans le capteur présenté sur la figure 1. Deux exemples d'applications présentant la passivation de l'acier et la dégradation d'un revêtement en époxy sont proposés. Dans une dernière partie, nous adapterons le capteur sensible à $E_{\text {corr }}$ pour réaliser un capteur de courant cathodique.

\section{Méthode et instrumentation}

L'élément sensible au potentiel $E_{\text {corr }}$ est présenté sur la figure 2. II est réalisé à partir d'un résonateur microruban stub sur un substrat FR4 $\left(\varepsilon_{r}=4.6\right)$. L'adaptation du circuit à $50 \mathrm{Ohm}$ a été obtenue en considérant une épaisseur du FR4 de $0.8 \mathrm{~mm}$ et une largeur de ligne de 1.6 $\mathrm{mm}$. La fréquence de résonance du stub est de $1.94 \mathrm{GHz}$ (stub avant varactor) pour une longueur de $1.8 \mathrm{~cm}$. Afin d'associer une variation de fréquence à une variation du potentiel $E_{\text {corr, }}$ une diode varactor (SMV1249) a été placée en bout de stub. Elle peut être assimilée à un condensateur, dont la capacité dépend de la différence de potentiel entre les électrodes 1 et 2. Outre la diode, un circuit de découplage $\mathrm{DC} / \mathrm{RF}$ a été réalisé. Les valeurs des composants utilisés sont $\mathrm{C}_{1}=100 \mathrm{pF} ; \mathrm{C}_{2}=47 \mathrm{nF}$ et L $=47 \mathrm{nH}$. L'élément sensible a été conçu pour présenter une résonance « moyenne » à $433 \mathrm{MHz}$ en vue d'une utilisation future du capteur dans cette bande ISM.
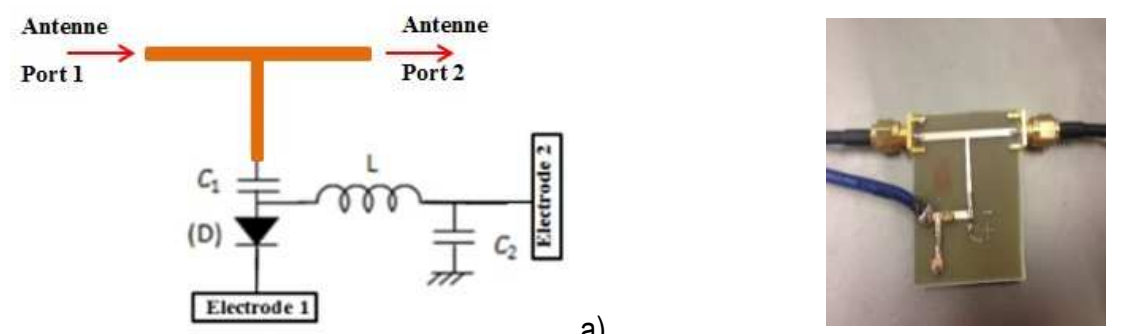

b)

Figure 2: (a) Schéma du capteur, le résonateur est représenté en orange (b) photographie du capteur

Le potentiel de corrosion Ecorr correspond au potentiel que prend un métal ou un alliage métallique dans un électrolyte donné par rapport à une électrode de référence. Dans cette étude, l'électrode 2 est l'électrode de référence (en zinc ou en calomel saturé). Deux types d'électrodes de travail (électrode 1) seront utilisés dans la suite : une électrode en acier et 
une électrode en acier recouverte d'une couche de peinture du type époxy. La mesure de $E_{\text {corr }}$ nécessite que les électrodes 1 et 2 (figure 2(a)) soient immergées dans un électrolyte, ici l'eau de mer. Cependant pour un bon fonctionnement du capteur, il est impératif que la propagation des ondes RF ne soit pas perturbée dans le reste du capteur. De ce fait, la partie présentée sur la figure 2(b) doit être protégée de l'eau par de la mousse, par exemple.

Afin de caractériser les variations fréquentielles du résonateur, deux méthodes ont été choisies. Elles consistent en l'utilisation d'un analyseur de réseaux (HP 8720B) et d'un module USRP N200 (ETTUS) associé à une carte WBX fonctionnant entre $50 \mathrm{MHz}$ et 2200 $\mathrm{MHz}$. Dans ce dernier cas, le dispositif de mesure est nettement moins encombrant et peut être facilement déplacé sur des lieux de mesures. Les résultats expérimentaux présentés ici ont été obtenus par la connexion des ports 1 et 2 à ces deux types d'instruments de mesure. Pour une utilisation en situation réelle, comme présenté sur la figure 1, des antennes réceptrice et émettrice doivent être insérées au niveau de ces deux ports.

\section{Exemples : passivation de l'acier et dégradation de peinture}

Afin de tester la faisabilité du capteur, la corrosion de l'acier inoxydable $316 \mathrm{~L}$ a été suivie dans l'eau de mer. Nous présentons sur la figure 3(a) l'évolution du potentiel de corrosion de l'acier $E_{\text {corr }}$ par rapport à une électrode en calomel saturé $\mathrm{Hg} / \mathrm{Hg}_{2} \mathrm{Cl}_{2}$ mesurée par un voltmètre. Comme le montre cette figure, l'acier se passive dans l'eau de mer grâce à la création de produits de corrosion à sa surface, entrainant ainsi une augmentation du potentiel $E_{\text {corr. }}$ Cependant, la variation de potentiel entre -0.3 et $0 \mathrm{~V}$ ne correspond pas à une plage de variation acceptable pour la polarisation du varactor. Pour cela, une électrode de référence en zinc a été choisie pour le capteur décrit sur la figure 2. Sur le graphe 3(b), nous présentons la transmission radiofréquence (paramètre $S_{12}$ ) du résonateur ainsi que la valeur du potentiel $E_{\text {corr }}$ de l'acier par rapport à cette nouvelle électrode de référence pour différents temps de corrosion. Comme attendu, l'augmentation de $E_{\text {corr }}$ de 600 à $950 \mathrm{mV}$ se traduit par un changement de fréquence du résonateur de 410 à $445 \mathrm{MHz}$. Les valeurs observées pour le potentiel $E_{\text {corr }}$ s'expliquent parfaitement. En effet, le potentiel électrochimique du zinc est de - $980 \mathrm{mV}$ et celui de l'acier de - $400 \mathrm{mV}$. Lorsque les deux matériaux sont placés dans la solution, la différence de potentiel entre les deux matériaux est donc, avant corrosion, d'environ $600 \mathrm{mV}$. Ce potentiel augmente ensuite comme nous l'avons expliqué précédemment du fait de la passivation de la surface.
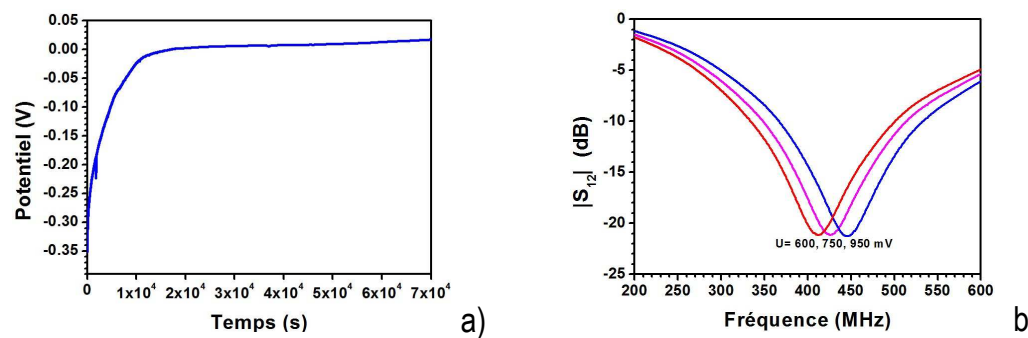

Figure 3: (a) Mesure du potentiel $E_{\text {corr }}$ en fonction du temps montrant la passivation de l'acier dans l'eau de mer et (b) le décalage fréquentiel du résonateur dû à la variation du potentiel de corrosion de l'acier par rapport à une électrode de référence en zinc 
Dans un second exemple, nous considérons le contrôle de la dégradation d'un revêtement époxy d'épaisseur environ $20 \mu \mathrm{m}$ sur de l'acier plongé dans l'eau de mer. Pour cela, la transmission du résonateur a été mesurée uniquement aux deux fréquences 415 et $480 \mathrm{MHz}$. Ce mode opératoire permet de s'affranchir de l'utilisation d'un analyseur de réseau. Nous présentons sur la figure 4 , les paramètres $S_{12}$ de transmission mesurés en fonction du temps. Afin de tester rapidement la dégradation de la peinture, une érosion mécanique lui a été appliquée. Comme attendu, durant les premières heures, la transmission RF reste stable aux deux fréquences. Au bout de quelques heures, du fait de la dégradation de la peinture et de l'intrusion de l'eau salée dans le revêtement, une variation de $E_{\text {corr }}$ entrainant un décalage fréquentiel de la résonance est observée. Ce changement se traduit par des variations de niveaux de transmission aux deux fréquences considérées, comme le montre la figure 4.

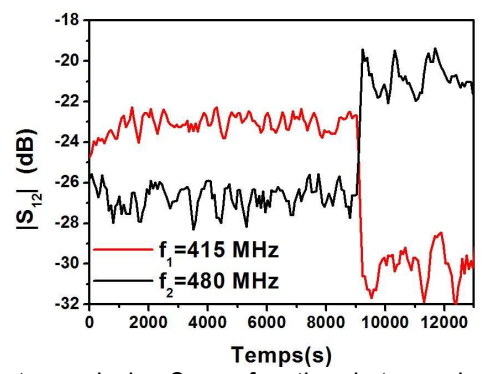

Figure 4: Variation du paramètre de transmission $S_{21}$ en fonction du temps lorsque l'acier est revêtu de la peinture

\section{Adaptation à la mesure de courants}

Une seconde version du capteur prévoit l'utilisation de 3 électrodes afin de réaliser simultanément la mesure de courants et tensions. Dans ce cas, le schéma du capteur est présenté sur la figure 5 . Deux résonateurs sont considérés. De la même façon que précédemment, une différence de potentielle est mesurée entre les électrodes 1 et 2 . Le second résonateur permet via l'utilisation de $\mathrm{R}_{\text {shunt }}$ de déterminer le courant circulant entre l'électrode 1 et 3. L'application principale de ce type de capteur est le monitoring des courants et tensions lors de l'utilisation de protection cathodique c'est-à-dire comprenant une anode sacrificielle, une électrode métallique à protéger.

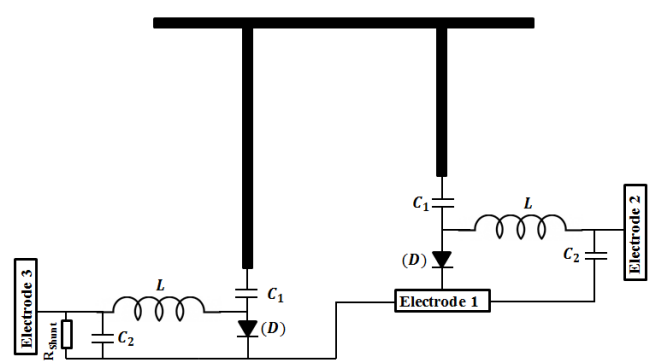

Figure 5: Schéma du capteur conçu pour la mesure du courant cathodique 


\section{Conclusion}

En conclusion, un élément sensible au potentiel de corrosion $E_{\text {corr }}$ a été développé en vue d'une intégration dans une architecture RFID « Chipless ». Différents exemples prouvant la faisabilité de la méthode ont été présentés. Le capteur proposé est peu encombrant, peu coûteux et totalement passif d'un point de vue énergétique. II peut, de ce fait, être placé dans des endroits où l'utilisation d'une batterie n'est pas possible. L'élément sensible développé ici doit être associé à un lecteur permettant la transmission et la réception du signal. Dans ce contexte, le point critique concerne l'isolation des ports TX et RX du lecteur. L'utilisation d'antennes à forts gains et possédant une bonne isolation peut être réalisée. Une distance de lecture de l'ordre du mètre peut alors être obtenue.

\section{Remerciements} thèse.

Un des auteurs (RK) remercie la Région Bretagne pour une contribution financière à sa

\section{Bibliographie}

[1] Srinivasan R, Carkhuff B.G, Phillips T.E, Saffarian H, Davis G.D, Raghu S. Miniature Wireless Full Spectrum EIS Corrosion Sensor. 2005.

[2] Davis G.D, Dunn R.C, Ross R.A. Wireless battery powered coated health monitor. Nace international, corrosion conference \& expo. 2010. p.10091.

[3] Wilson R, Muscat R.F. Novel thin wire paint and sealant degradation sensor. Sensors and Actuators A: Physical. 2011.169. p.301-307.

[4] SeonYeob L, Young-Geun K, Sungwon J, Hong-Seok S, Seong-Min L. Application of steel thin film electrical resistance sensor for in situ corrosion monitoring. Sensors and Actuators B. 2007. 120. 368377.

[5] Dante J.F, Steiner A, Friedersdorf F, RF. System for corrosivity monitoring. Nace international, corrosion conference \& expo. 2008. p.08204.

[6] Materer N.F, Apblett A.W. Passive wireless corrosion sensor. US 2010. Patent 20090058427.

[7] Preradovic S, Karmakar N.C. Design of fully printable planar chipless RFID transponder with 35-bit data capacity. European Microwave Conference. 2009. p. 013-016.

[8] Yasri M, Gallee F, Lescop B, Diler E, Thierry D, Rioual S. Fundamental basis of electromagnetic wave propagation in a zinc microstrip lines during its corrosion. Sensors and Actuators B: Chem. 2016. 223. p. 352-358. 
[9] Yasri M, Gallee F, Lescop B, Diler E, Thierry D, Rioual S. Passive Wireless Sensor for Atmospheric Corrosion Monitoring. Proceedings of the 8th European conference on Antennas and Propagation. EUCAP, IEEE. 2014. p.2945-2949.

[10] Rammal J, Delhote N, Tantot O, Verdeyme S, Gallee F, Rioual S. New passive ceramic sensor for corrosion detection. Proceedings of the 44rd European Microwave Conference. European Microwave Conference, IEEE. 2014. p.167-170.

[11] Landoulsi J, Elkirat K, Richard C, Feron D, Pulvin S. Enzymatic Approach in Microbial-Influenced Corrosion: A review Based on Stainless Steels in Natural Waters. Environmental Science \& Technology. 2008. 42. p.2233-2242. 